\title{
A case report and literature review of primary resistant Hodgkin lymphoma: a response to anti-PD-I after failure of autologous stem cell transplantation and brentuximab vedotin
}

\author{
This article was published in the following Dove Press journal: \\ OncoTargets and Therapy \\ 21 September 2016 \\ Number of times this article has been viewed
}

Peipei Xu

Fan Wang

Chaoyang Guan

Jian Ouyang

Xiaoyan Shao

Bing Chen

Department of Hematology, The Affiliated Drum Tower Hospital of Nanjing University Medical School,

Nanjing, People's Republic of China
Correspondence: Bing Chen;

Xiaoyan Shao

Department of Hematology, The Affiliated

Drum Tower Hospital of Nanjing

University Medical School, Nanjing 210008 ,

People's Republic of China

Tel +86258310 521I

Email chenb21I@163.com;

15358184562@I63.com

\begin{abstract}
Hodgkin lymphoma (HL) is a highly curable hematologic malignancy, and $~ 70 \%$ of cases can be cured with combination chemotherapy with or without radiation. However, patients with primary resistant disease have a cure rate of $<30 \%$. For such patients, high-dose chemotherapy followed by autologous stem cell transplantation (ASCT) is considered to be the standard treatment. If patients fail to respond to ASCT or relapse soon thereafter, they usually receive another ASCT, allogeneic stem cell transplantation or treatment with novel agents. This case report presents the case of a 54-year-old patient with primary resistant HL who received single-agent treatment, brentuximab vedotin, after ASCT relapse. Despite treatment with brentuximab vedotin, the disease continued to progress. In patients with such highly resistant disease, the treatment options are limited. Depending on the physical condition and the willingness of the patient, pembrolizumab, a programmed cell death protein-1 inhibitor, can be given as salvage therapy. But, out of our expectation, the patient achieved a very good partial response after four cycles of pembrolizumab. No serious adverse events were observed with pembrolizumab treatment. This case provides support for a new and effective strategy for treating primary resistant Hodgkin lymphoma.
\end{abstract}

Keywords: Hodgkin lymphoma, autologous stem cell transplant, brentuximab vedotin, pembrolizumab, PD-1, good response

\section{Background}

In recent decades, the cure rate of Hodgkin lymphoma (HL) has significantly increased because of the advances in combination chemotherapy and radiation therapy (RT). As a result, $>70 \%$ of newly diagnosed patients are cured. However, for patients with primary resistant disease, which is defined as disease that progresses during first-line therapy or within the first 3 months of achieving remission, the cure rate is $<30 \%$. ${ }^{1}$ High-dose chemotherapy (HDCT) followed by autologous stem cell transplantation (ASCT) is recommended as the standard treatment for patients with primary resistant disease..$^{2-4}$ In addition, RT can be used to treat limited and residual nodal site diseases and patients who have not previously received RT. Because of limited bone marrow reserve and more chemotherapy resistance, patients who relapse after ASCT have limited treatment options. These options include repeated ASCT, allogeneic stem cell transplantation (alloSCT), or treatment with brentuximab vedotin or other novel agent. Based on its promising results ( $75 \%$ response rate and $34 \%$ complete remission [CR]) from a phase II open-label study, ${ }^{5}$ brentuximab vedotin has been approved by the US Food and Drug 
Administration (FDA) and European Medicines Agency as a single agent for the treatment of HL in patients who have relapsed after ASCT. The patient in this case report experienced relief of clinical symptoms after three cycles of brentuximab vedotin; however, the disease progressed during the subsequent three cycles of treatment. In this setting, further treatment options were extremely limited.

The HL described in this report was characterized by malignant Hodgkin Reed-Sternberg cells surrounded by large numbers of $\mathrm{T}$ cells that lacked antitumor activity. Recent studies have demonstrated the activation of programmed cell death protein-1 (PD-1) signaling pathway, an efficient immunosuppressive mechanism, by which HL evades immune system. The results from several clinical trials in which $>60 \%$ of the participants had failed after ASCT and brentuximab vedotin treatment have suggested that a PD-1 immune checkpoint inhibitor is an effective and well-tolerated single agent for relapsed and refractory HL., Pembrolizumab, which is the first PD-1 immune checkpoint inhibitor to be approved by the FDA, has primarily been used to treat solid malignancies including melanoma and lung carcinoma. ${ }^{8}$ Pembrolizumab has also demonstrated remarkable antitumor activity in patients with heavily pretreated $\mathrm{HL}$ and has been approved in the US, Europe, Australia, and other countries and regions. However, there are few reports of pembrolizumab treatment in patients with heavily pretreated $\mathrm{HL}$ in Asia. Here, the present study reports the case of an Asian patient with primary resistant HL who relapsed after ASCT and brentuximab vedotin treatment and then unexpectedly achieved very good partial response (VGPR) following pembrolizumab treatment.

\section{Case presentation}

A 54-year-old female was diagnosed with classical HL (Figure 1) involving her neck, mediastinal, and left hilus pulmonis lymph nodes corresponding to stage IIB (fever) in May 2012. According to the German Hodgkin Study Group classification system, the patient was considered to have advanced $\mathrm{HL}$ as the erythrocyte sedimentation rate was $>30 \mathrm{~mm} / \mathrm{h}$ with B symptoms and more than two regional lymph nodes were invaded, without extranodal involvement as assessed by positron emission tomography (PET)/computed tomography (CT) (Figure 2). On the basis of the National Comprehensive Cancer Network clinical guidelines for the treatment of HL, the patient received four cycles of ABVD (doxorubicin, bleomycin, vincristine, and dacarbazine). Following ABVD treatment, the patient experienced relief of clinical symptoms (fever and cough). In addition, the neck and right hilus pulmonis lymph nodes had decreased in size. Although the mediastinal lymph nodes had also diminished, PET/CT revealed that their metabolic activity had not decreased obviously or detectably (Figure 2). Moreover, PET/CT also identified that the seventh right rib was a new zone of increased metabolism compared with the initial scan.

As the patient achieved a partial response (PR) after four cycles of ABVD, she received two cycles of IGVE (cyclophosphamide, gemcitabine, vinorelbine, and prednisone) beginning in November 2012. In January 2013, examination by PET/CT demonstrated negative metabolic activity of the neck and left hilus pulmonis lymph nodes, and seventh right rib. However, the lymph nodes anterior to the aortic arch demonstrated residual high metabolic activity. The patient received another third cycle of IGVE, followed by ASCT. In May 2013, according to a PET/CT examination, the patient received radiotherapy with 36 Gy extendedfield radiation plus another 18 Gy directed to the involved tissues. Less than 3 months after completing radiotherapy, the patient again suffered from fever and cough. Examination by PET/CT demonstrated multifocal progressive disease involving primary and multiple bilateral pulmonary lesions with increased metabolism of different levels compared with the previous PET/CT scan. Considering that the disease had relapsed and progressed, a new histologic analysis was warranted to eliminate the possibility of a second tumor or other benign diseases. Due to percutaneous lung centesis in the left pulmonary lesions and limited biopsy tissue, histopathology and immunohistochemistry were insufficient to provide a definitive diagnosis. However, it was confirmed that lymphocytic invasion of the pulmonary lesions had occurred, and some large cells stained positive for CD30 and CD15. These results indicated that the disease had progressed to stage IV B (fever). According to the Spanish Society for Medical Oncology clinical guidelines, brentuximab vedotin, a CD30 antibody, is the preferred option for HL patients who have relapsed after ASCT. As a bridge to receive brentuximab vedotin, the patient received one cycle of ICE (etoposide, cyclophosphamide, and carboplatin). In October 2013, the patient commenced therapy with brentuximab vedotin $(100 \mathrm{mg} \times 1$ day, every 3 weeks). After three cycles, her clinical symptoms (fever and cough) were relieved, but no significant changes were observed on imaging tests.

In March 2014, the patient received an additional three cycles of brentuximab vedotin and was readmitted to the hospital with fever, cough, bloody sputum, and unexplained weight loss. In order to determine whether multiple bilateral pulmonary lesions were involved, the patient underwent 

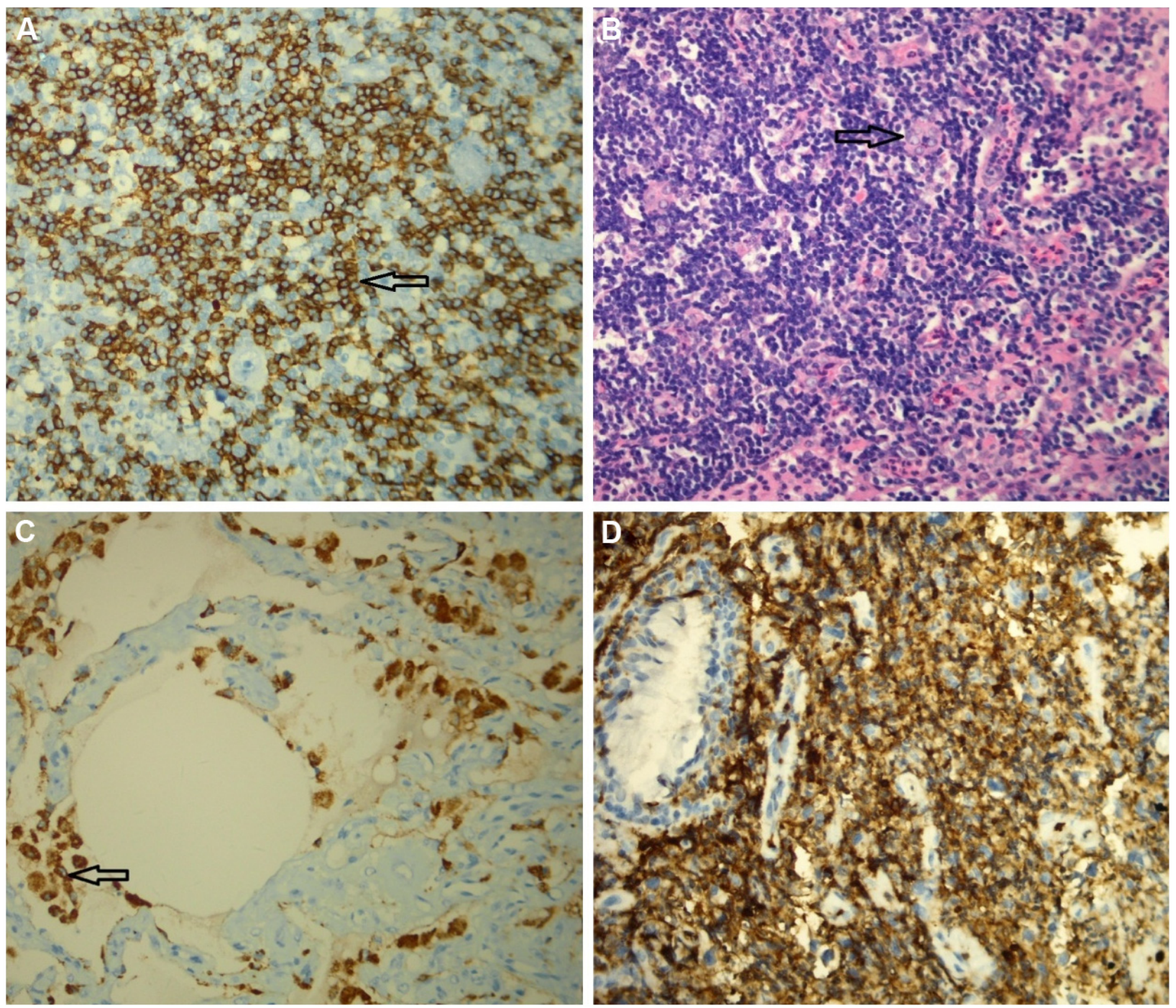

Figure I Pathology and immunohistochemistry.

Notes: (A) The arrow points to Hodgkin lymphoma cells expressing CD30. The image was obtained by staining CD30 when the disease was diagnosed in May 20I2. (B) The arrow points to Hodgkin Reed-Sternberg cells surrounded by a large number of inflammatory and immune cells in an involved lymph node. The image was obtained from the initial involved lymph node. (C) The arrow points to the large cells expressing CD30 admixed with lymphocyte cells. These larger cells also expressed CDI5, but we could not exclude the possibility of lung involvement. However, the patient exhibited classical B symptoms and experienced recurrence with incomplete remission. According to the PET/CT examination, the disease was classified as stage IV B lymphoma in September 20I3. (D) After ASCT failure, the patient received six cycles of brentuximab vedotin treatment. However, the disease progressed after these therapies in April 20I4. Then, through fiberoptic bronchoscopy lesion biopsy, the patient's multiple bilateral pulmonary lesions were diagnosed with involved classical Hodgkin lymphoma cells. Magnification (A and B) is $40 \times 10$ and (C and D) is $100 \times 10$.

Abbreviations: ASCT, autologous stem cell transplantation; CT, computed tomography; PET, positron emission tomography.

fiberoptic bronchoscopy lesion biopsy and was diagnosed with classical HL. The CT scan demonstrated bilateral pulmonary obstructive pneumonia, and the patient was in a poor physical condition. Following this diagnosis, she received antiinfection and support treatment and initiated treatment with intermittent low-dose combination chemotherapy (prednisone $20 \mathrm{mg}$, cyclophosphamide tablets $100 \mathrm{mg}$, and etoposide capsules $50 \mathrm{mg}$ ) beginning in June 2014. Four months later, multiple enlarged abdominal lymph nodes were observed on a CT scan, indicating that the disease was still progressing. At that point, alloSCT was deemed to be the most appropriate therapy. However, the patient was in poor physical condition and was unable to stand. More importantly, she refused to undergo alloSCT. To control disease progression, she was treated with two cycles of MOAP (mechlorethamine, vincristine, procarbazine, and prednisone) and one cycle of mechlorethamine with dexamethasone. The general condition of the patient continued to deteriorate, and she experienced fever, cough, pruritus, fatigue, and weight loss.

Because of its remarkable antitumor activity and relatively mild toxicities, pembrolizumab, an immunoglobulin G4 (IgG4) monoclonal antibody against PD-1, is regarded as an effective salvage therapy for heavily pretreated HL patients, 

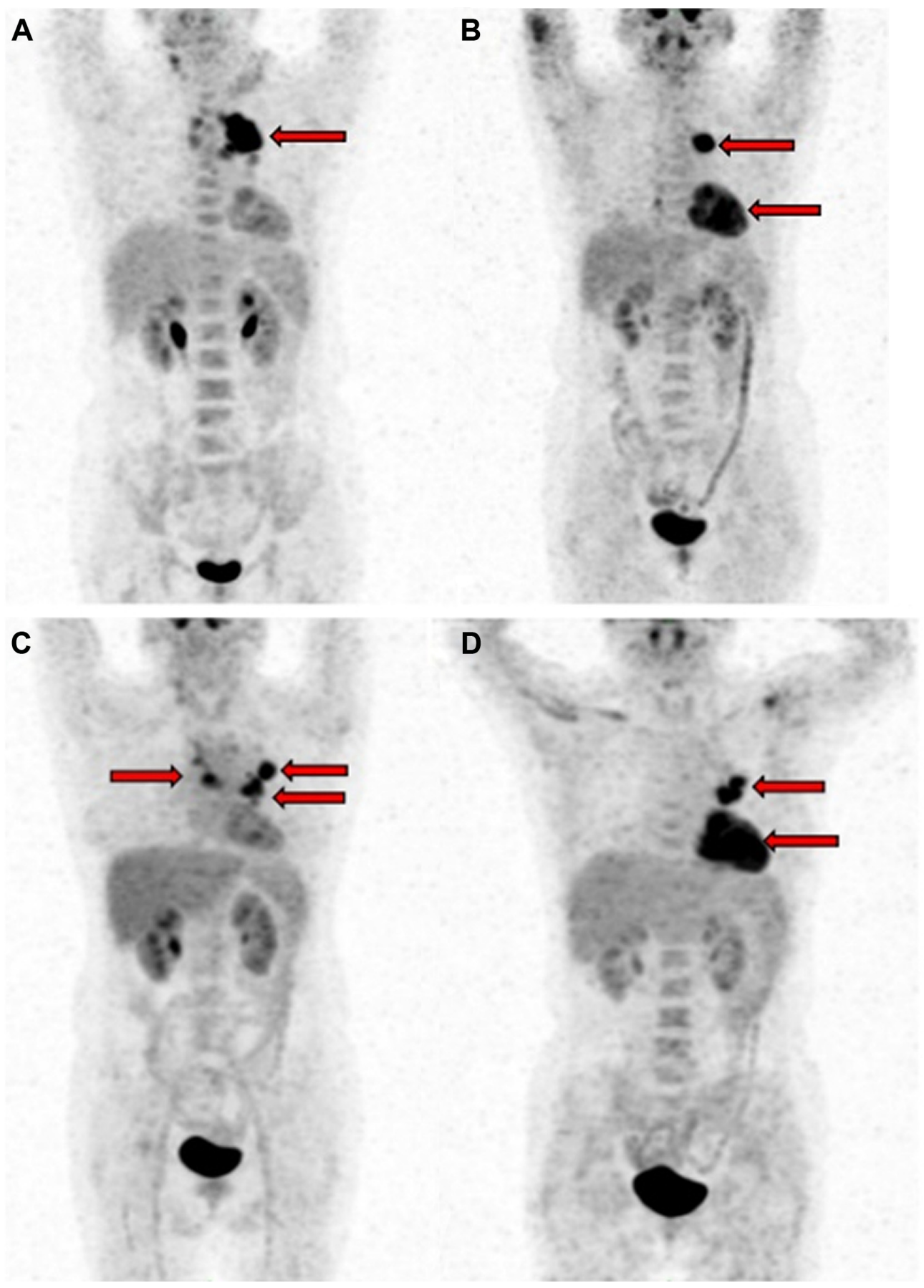

Figure 2 Response to therapy as shown on the PET/CT.

Notes: (A) The patient was diagnosed with classical Hodgkin lymphoma (HL) involving the neck, mediastinal and left hilus pulmonis lymph nodes in stage II B (fever) disease in May 2012. (B) After four cycles of ABVD, the neck and right hilus pulmonis lymph nodes faded. The mediastinal lymph nodes diminished but still appeared metabolically active in October 2012. Furthermore, the disease involved a new lesion. (C) After ASCT in May 20I3, a partial response was achieved, and the mediastinal lymph nodes remained as residual lesions. (D) After the patient received ASCT and radiotherapy, the patient suffered again from fever and cough. The PET/CT demonstrated multifocal progressive disease involving primary and additional involved lymph nodes with increased metabolism of different levels compared with the previous PET/CT scan. The arrows indicate mediastinal and hilus pulmonis lymph nodes involved with Hodgkin lymphoma cells.

Abbreviations: ABVD, doxorubicin, bleomycin, vincristine, and dacarbazine; ASCT, autologous stem cell transplantation; CT, computed tomography; PET, positron emission tomography.

particularly those who have failed treatment with ASCT and brentuximab vedotin. With the consent of the patient, she received single-agent pembrolizumab (Merck \& Co., Inc., Whitehouse Station, NJ, USA) at $2 \mathrm{mg} / \mathrm{kg}$ (100 mg day 1) every 3 weeks. During the initial two cycles of pembrolizumab at a dose of $2 \mathrm{mg} / \mathrm{kg}$, the patient experienced severe inflammation reaction, and the main manifestations were persistent high fever, cough, and hypotension. Considering the presence of mediastinal and hilus pulmonis lymph nodes and multiple bilateral pulmonary lesions and that the patient was heavily 
pretreated, the dose was reduced to $1 \mathrm{mg} / \mathrm{kg}$ in subsequent cycles to decrease the drug-related risk. Nevertheless, after two cycles of pembrolizumab, the patient experienced relief of clinical symptoms, and her clinical conditions improved. Examination with a CT scan following an additional two cycles of treatment revealed a drastic decrease in the size of both the lesions, a response that was classified as PR. After six cycles of treatment for pneumonitis, she achieved VGPR. Until June 2016, the patient received 12 cycles of pembrolizumab, and she still achieved benefit from this single-agent treatment. In this case, treatment-related adverse events (AEs), including transient fever after infusion, loss of appetite, fatigue, pruritus, and dizziness, were tolerated.

\section{Discussion}

$\mathrm{HL}$ is a highly curable hematologic malignancy treated with the combination chemotherapy with or without RT. ABVD is the preferred first-line combination chemotherapy for HL. Following treatment with 6-8 cycles of ABVD, $>70 \%$ of patients are considered to be cured. However, conventional dose chemotherapy is not sufficient to cure refractory or early relapsing cases. HDCT followed by an ASCT is recommended as the standard treatment for those who relapse after initial therapy. If this approach fails, most patients can choose to receive a second ASCT, alloSCT or treatment with novel agents such as brentuximab vedotin, the preferred option for patients who relapse after ASCT. Brentuximab vedotin is a CD30 antibody conjugated to CD30 and gets internalized by attaching to monomethyl auristatin E causing microtubule disruption that results in cell cycle arrest at M phase. ${ }^{9}$ Brentuximab vedotin is associated with a $>60 \%$ overall response rate and $\mathrm{CR}$ in $\sim 30 \%$ of cases of relapsed and refractory HL. Based on these findings, the patient in this report received brentuximab vedotin after ASCT failure. After three cycles of brentuximab vedotin, the patient's clinical symptoms were relieved. The response period of three cycles is consistent with the observations from several clinical studies. However, further treatment with brentuximab vedotin did not halt disease progression.

As previously mentioned, HL was characterized by the presence of malignant Hodgkin Reed-Sternberg cells admixed with inflammatory and immune cells that lacked antitumor activity. One of the mechanisms by which ReedSternberg cells evade immunity is by overexpressing PD-1 ligands (PD-L1/B7-H1 and PD-L2/B7-DC), thereby activating the PD-1 signaling pathway, which results in decreased proliferation, inhibiting antitumor activity, and increasing apoptosis of cytotoxic T cells. ${ }^{10}$ In addition, PD- 1 can attenuate the immune response by promoting proliferation of regulatory T cells. ${ }^{11}$ Thus, blocking the PD-1 signaling pathway can effectively increase antitumor immunity. In September 2014, pembrolizumab became the first anti-PD-1 agent approved by the FDA and has mainly been used for the treatment of solid tumors, such as unresectable or metastatic melanoma and non-small-cell lung cancer.

Because of the successful results observed in other cancers, pembrolizumab has also been used to treat relapsing and refractory HL in recent years. After treatment failure with ASCT and brentuximab vedotin, the patient in this report had limited options, and she received several salvage combination chemotherapies to control disease progression. In this highly refractory case of HL, after two cycles of pembrolizumab, the patient's clinical symptoms were relieved, and her clinical condition improved. After four cycles of treatment, both the lesions decreased in size. Through six cycles of pembrolizumab, she achieved VGPR and remained in remission for 14 months after initial treatment. This response to pembrolizumab was unexpected.

In a phase IB trial, patients aged 20-67 years (median age 32 years) with several hematologic malignancies including relapsed and refractory HL were treated with pembrolizumab at a dose of $10 \mathrm{mg} / \mathrm{kg}$ every 2 weeks. ${ }^{12}$ Among these 31 patients with relapsed and refractory HL after failure of brentuximab vedotin treatment, five patients achieved CR, 15 achieved PR, and seven had stable disease, and the overall response rate was $65 \%$. Although, there were no grade 4 AEs or fatal events related to this immunotherapy, AEs of any other grade were noted in 30 of the 31 patients, and five patients experienced grade 3 drug-related AEs. On the basis of Eastern Cooperative Oncology Group performance status $<2$ as inclusion criteria and active or past documented autoimmune disease, evidence of interstitial lung disease, or HIV infection as the exclusion criteria. In another report, two European patients with refractory HL were treated with pembrolizumab at a low dose of $2 \mathrm{mg} / \mathrm{kg}$ every 3 weeks and achieved at least near-CR after three cycles of this single-agent therapy. ${ }^{13}$ One of the patients, a 21-year-old male, with stage IV HL, achieved CR after first six cycles of brentuximab vedotin, but relapsed 4 months later after ASCT, and retreatment was ineffective. The other patient was a 38 -year-old female with stage III HL, without response to brentuximab vedotin. After initial treatment of pembrolizumab, both of them remained in remission for $>20$ weeks and no AEs were observed. In this report, the patient received a dose of pembrolizumab that was much lower at $1 \mathrm{mg} / \mathrm{kg}$ every 3 weeks ( $2 \mathrm{mg} / \mathrm{kg}$ at initial two cycles) than what was used in aforementioned research $(2-10 \mathrm{mg} / \mathrm{kg}$ every 2-3 weeks); however, it was still effective and safe. As 
distinct from rituximab, a chimeric murine/human $\mathrm{mAb}$ binding to CD20-positive B cells, pembrolizumab is a humanized IgG4 anti-PD-1 mAb. The limited Fc receptor engagement of pembrolizumab leads to its antitumor effect independent of antibody-dependent cell-modulated cytotoxicity of PD-1-positive cells, but enhancement of cytotoxic T-cell activity and suppression of regulatory T-cell function. ${ }^{14}$ In this case, increased cytotoxic $\mathrm{T}$ cells and subdued regulatory $\mathrm{T}$ cells result in a decrease in peripheral tolerance to auto-antigens and recapitulate elements of autoimmunity in different organs both of which contribute to serious drugrelated AEs. ${ }^{15}$ During the first two cycles of pembrolizumab, the patient experienced severe inflammation reaction, including transient fever after infusion, loss of appetite, fatigue, pruritus, and dizziness. These symptoms were alleviated after the appropriate treatments and did not return. However, it is possible that this condition resulted from the patient being heavily pretreated, having limited bone marrow reserve, experiencing immunosuppression, and being in poor clinical condition. Mediastinal and hilus pulmonis lymph nodes and multiple bilateral pulmonary lesions and obstructive pneumonitis caused by tumor compression can also increase the risk of infection. Before treatment with pembrolizumab, the patient had suffered from severe pneumonia which was observed on the CT scans. During the initial two cycles of pembrolizumab, the patient's clinical symptoms were relieved gradually, and pneumonitis never got worse when observed in CT scans. Above all, the pneumonitis of this patient was a priori considered as disease-related. At a much lower dose of pembrolizumab, the high-risk patient achieved high and persistent response for 14 months without severe drug-related AEs. In order to find optimal dose to minimize AEs, further research of low-dose pembrolizumab is needed.

In previously reported clinical trials, increased lactate dehydrogenase (LDH) levels during the treatment with pembrolizumab were reported in $\sim 30 \%$ of patients. ${ }^{16,17}$ In HL, LDH testing is a mandatory laboratory test as it is a factor associated with disease staging, prognosis, and response. However, the patient's LDH level in this report was mainly in the normal range (Figure 3 ). As pembrolizumab promotes cytotoxic T-cell activity and suppresses the function of regulatory T cells, the lymphocyte subgroups of the patient's peripheral blood were dynamically monitored. During treatment with pembrolizumab, the levels of $\mathrm{CD}^{+} \mathrm{T}$ cells rapidly increased and a peak in levels was observed after the initial two cycles ( $2 \mathrm{mg} / \mathrm{kg}$ every 3 weeks) (Figure 4$)$. During that time, the patient experienced relief of clinical symptoms. Due to a case of severe pneumonitis, the dose was

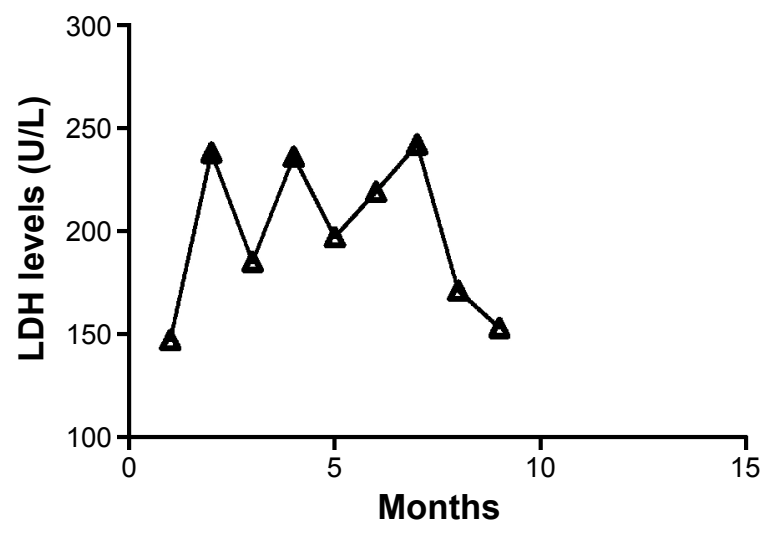

Figure 3 The patient's LDH level before and during treatment with pembrolizumab. Notes: In previously reported clinical trials, approximately $30 \%$ of patients had elevated LDH levels during pembrolizumab treatment. In Hodgkin lymphoma, LDH testing is a factor associated with disease staging, prognosis, and response. The LDH levels in this patient were primarily within the normal range (mean value was 205.12 U/L). Abbreviation: LDH, lactate dehydrogenase.

reduced to $1 \mathrm{mg} / \mathrm{kg}$ every 3 weeks for subsequent treatments. Unexpectedly, after two cycles at $2 \mathrm{mg} / \mathrm{kg}$ and two cycles at $1 \mathrm{mg} / \mathrm{kg}$, the $\mathrm{CD}^{+} \mathrm{T}$-cell level did not decrease (Figure 4). In addition, the total tumor burden was dramatically reduced (Figure 5). Following six cycles of pembrolizumab, the patient achieved VGPR. During this period, cytotoxic T cells reached another peak that was higher than the previous one (Figure 4). In this case, the increased level of $\mathrm{CD}^{+} \mathrm{T}$ cells correlated with therapeutic efficacy.

Tumor infiltrating lymphocyte (TIL) is known as the lymphocyte around the tumor cells. Because of the close

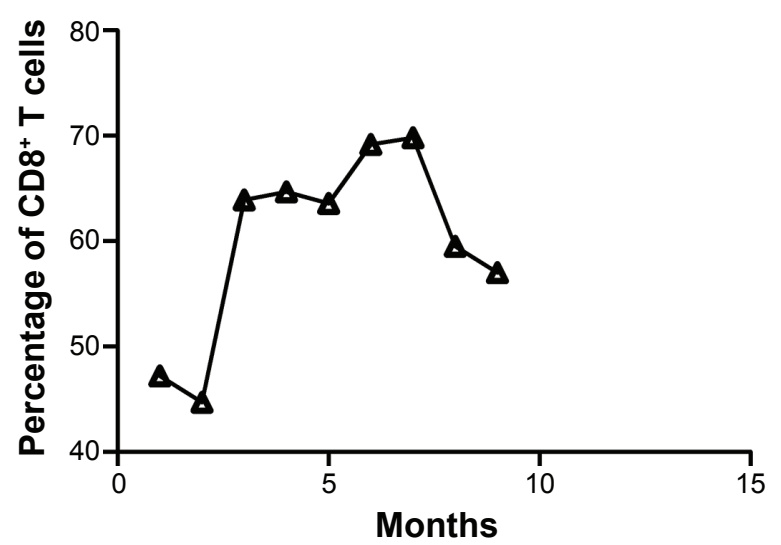

Figure 4 The percentage of $C D 8^{+} \mathrm{T}$ cells in lymphocyte subgroups at peripheral blood before and during treatment with pembrolizumab.

Notes: During treatment with pembrolizumab, $C D 8^{+} \mathrm{T}$ cell levels rapidly increased and reached an initial peak after two cycles $(2 \mathrm{mg} / \mathrm{kg}$ every 3 weeks). At that time, the patient's clinical symptoms were relieved. As the patient was diagnosed with serious pneumonitis, the dose of subsequent treatments was reduced to $1 \mathrm{mg} / \mathrm{kg}$ every 3 weeks. Unexpectedly, after two cycles at $2 \mathrm{mg} / \mathrm{kg}$ and two cycles at $1 \mathrm{mg} / \mathrm{kg}$, the patient's high level of $\mathrm{CD}^{+} \mathrm{T}$ cells was maintained. During that time, there was a dramatic reduction in total tumor burden. After she received six cycles of pembrolizumab, the patient achieved VGPR. During this period, the patient's cytotoxic $T$ cell levels reached another peak, which was higher than the previous one. Abbreviation: VGPR, very good partial response. 

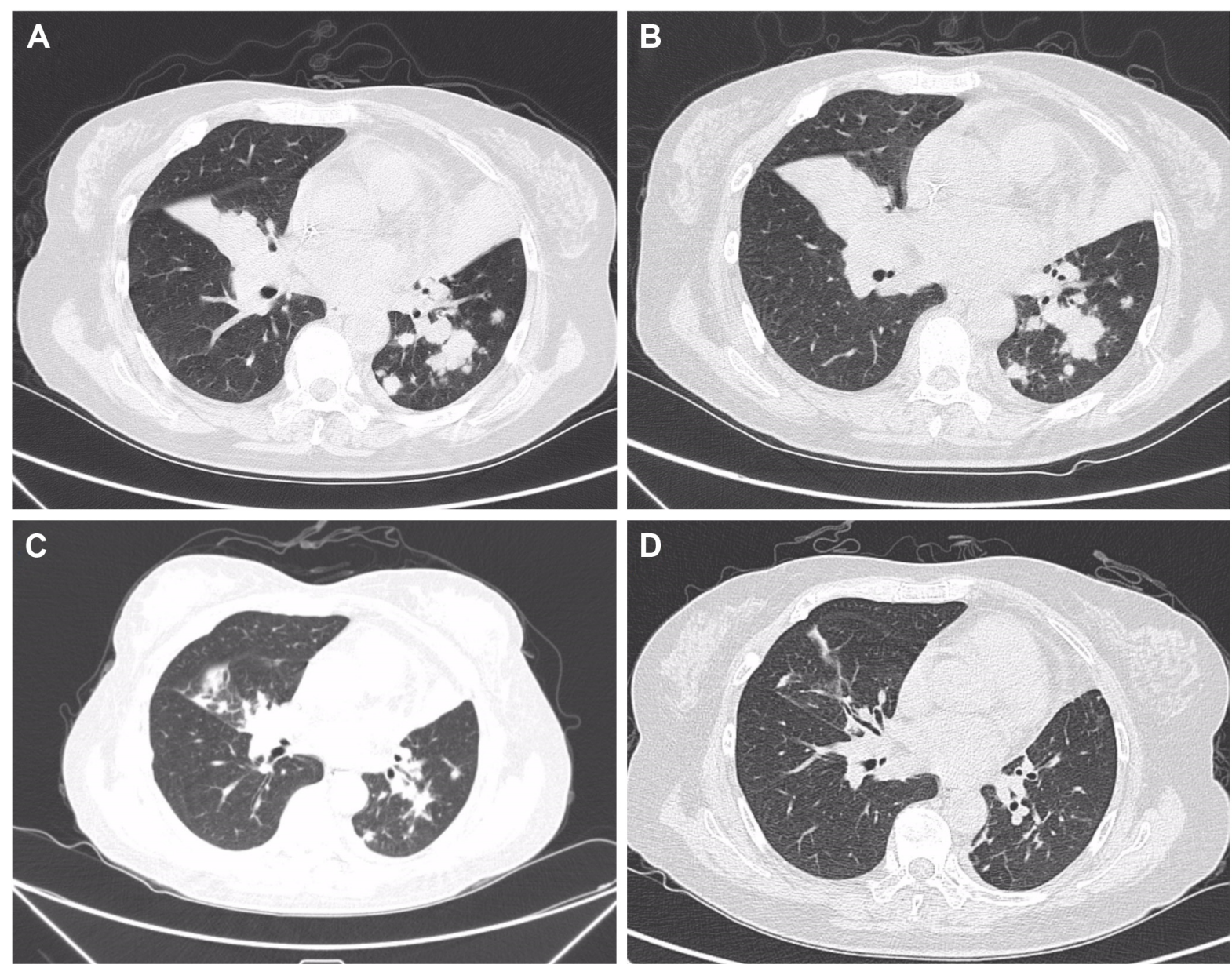

Figure 5 Tumor burden, as assessed by CT scans, before and during the treatment with pembrolizumab.

Notes: (A) Before treatment with pembrolizumab, mediastinal, and hilus pulmonis lymph nodes, multiple bilateral pulmonary lesions and bilateral pulmonary obstructive pneumonia were observed on the CT scans in February 2015. At that time, the patient's general condition was worsening, and she was experiencing fever, cough, pruritus, fatigue, and weight loss. (B) After two cycles of pembrolizumab at $2 \mathrm{mg} / \mathrm{kg}$ every 3 weeks, the patient's clinical symptoms were relieved, but there were no significant changes observed in CT scans in April 20I5. (C) After two cycles at $2 \mathrm{mg} / \mathrm{kg}$ and two cycles at I mg/kg, the patient's tumor burden was dramatically reduced in June 20I5. (D) After six cycles of pembrolizumab, the patient achieved VGPR in October 2015.

Abbreviations: CT, computed tomography; VGPR, very good partial response.

contact with tumor cells, TIL is considered as the specific and direct response of antitumor immunity. A large number of the TILs with normal function symbolize the strong antitumor activity of the host. However, in fact, most of these TILs are immunosuppressive and anergic induced by various cytokines and chemokines secreted by tumor cells. Malignant cells express the PD-1 ligands to bind PD-1 which is expressed on the surface of various immune cells, to downstream kinase signaling inhibition including the PI3K pathway, and ultimately suppression of immune cells function in the tumor microenvironment. ${ }^{10}$ In solid tumors, TIL mainly consists of $\mathrm{CD} 8^{+} \mathrm{T}$ cells which play a significant role in antitumor immunity. ${ }^{18}$ However, in this patient, the TIL is mainly composed of $\mathrm{CD}^{+}{ }^{+} \mathrm{T}$ cells which are known as regulatory $\mathrm{T}$ cells and suppress the function of cytotoxic $\mathrm{T}$ cells. With interspersed $\mathrm{CD} 8^{+} \mathrm{T}$ cells as the background infiltrate cells, malignant Hodgkin Reed-Sternberg cells are surrounded by these $\mathrm{CD}^{+} \mathrm{T}$ cells (Figure 6). Recently, several vital clinical trials ${ }^{17,19,20}$ demonstrate whether expression and the level of PD-L1 in tumor cells are considered as the potential predictive factors for the treatment response to PD-1 inhibitors. For this patient, in the initial involved lymph node, $\sim 40 \%$ of HL cells and $15 \%$ surrounding $\mathrm{CD}^{+} \mathrm{T}$ cells express PD-L1, but the background cells do not express. Therefore, the patient's antitumor immunity strengthened dramatically by blocking the PD-1 signaling pathway when treated with pembrolizumab. As previously mentioned, the levels of $\mathrm{CD}^{+} \mathrm{T}$ cells rapidly increased after treatment with pembrolizumab and stayed at a high level during the treatment.

In conclusion, pembrolizumab treatment was an effective, tolerable, and safe single agent for this patient with highly 

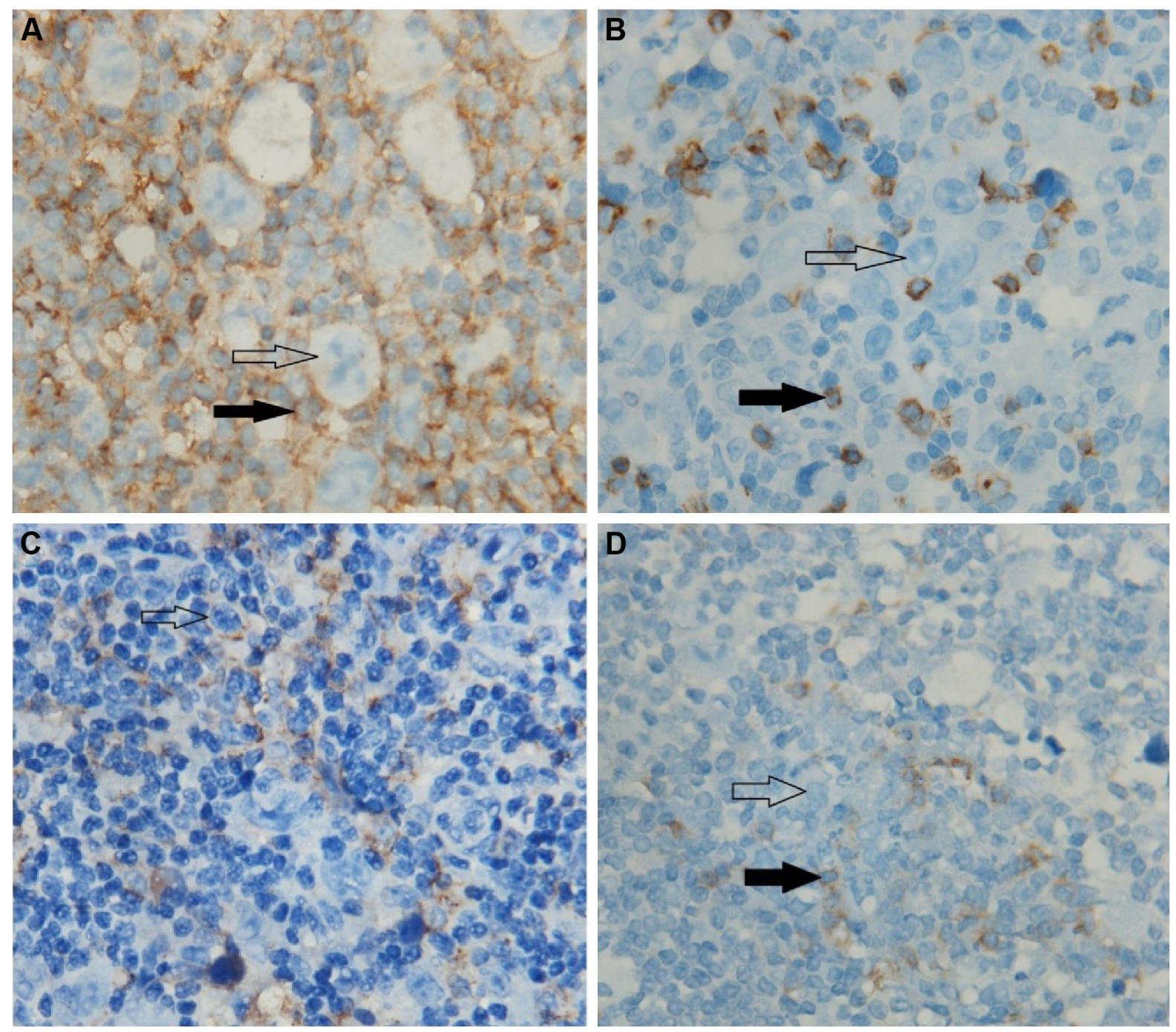

Figure 6 Immunohistochemistry (the initial involved lymph node).

Notes: (A) Malignant Hodgkin Reed-Sternberg cells were surrounded by $\mathrm{CD}^{+}{ }^{+} \mathrm{CD} 4^{+} \mathrm{T}$ cells. (B) Hodgkin Reed-Sternberg cells were interspersed with $\mathrm{CD} 3{ }^{+} \mathrm{CD} 8^{+} \mathrm{T}$ cells which are considered as the background infiltrate cells. (C) In the initial involved lymph node, nearly $40 \%$ of $\mathrm{HL}$ cells and $15 \%$ of surrounding CD4+ $\mathrm{T}$ cells expressed PD-LI, but the background cells did not express PD-LI. (D) The tumor cells and surrounding cells were negative for PD-I expression, and nearly 25\% of background cells were positive. The blank arrows indicate the Hodgkin lymphoma cells. The solid arrows indicate tumor infiltrating lymphocyte. Magnification is I00 10.

resistant HL, whose disease had progressed after treatment failure with ASCT and brentuximab vedotin. Furthermore, the expression of PD-L1 in HL cells, TIL status, and changes in the lymphocyte subgroups of the patient's peripheral blood should be taken into comprehensive consideration for the therapeutic effectiveness of HL patients.

\section{Acknowledgments}

We would like to thank the patient who took part in this study. Written informed consent for the publication of this case report and the accompanying images was obtained from the patient.

This work was supported by the National Natural Science Foundation of China (81400162 and 81570174), the Natural Science Foundation of Jiangsu Province (BK20140100), the Technique Development Foundation of Nan Jing (Outstanding Youth Foundation, JQX15004), and the Medical Science and Technology Development Program of Nanjing (Ykk14069 and Ykk14056).

\section{Disclosure}

The authors report no conflicts of interest in this work.

\section{References}

1. Provencio Pulla M, Alfaro Lizaso J, de la Cruz Merino L, et al. SEOM clinical guidelines for the treatment of follicular non-Hodgkin's lymphoma. Clin Transl Oncol. 2015;17(12):1014-1019.

2. Linch DC, Winfield D, Goldstone AH, et al. Dose intensification with autologous bone-marrow transplantation in relapsed and resistant Hodgkin's disease: results of a BNLI randomised trial. Lancet. 1993; 341(8852):1051-1054. 
3. Rancea M, von Tresckow B, Monsef I, Engert A, Skoetz N. High-dose chemotherapy followed by autologous stem cell transplantation for patients with relapsed or refractory Hodgkin lymphoma: a systematic review with meta-analysis. Crit Rev Oncol Hematol. 2014;92(1):1-10.

4. Schmitz N, Pfistner B, Sextro M, et al. Aggressive conventional chemotherapy compared with high-dose chemotherapy with autologous haemopoietic stem-cell transplantation for relapsed chemosensitive Hodgkin's disease: a randomised trial. Lancet. 2002;359(9323): 2065-2071.

5. Pro B, Advani R, Brice P, et al. Brentuximab vedotin (SGN-35) in patients with relapsed or refractory systemic anaplastic large-cell lymphoma: results of a phase II study. J Clin Oncol. 2012;30(18):2190-2196.

6. Ansell SM, Lesokhin AM, Borrello I, et al. PD-1 blockade with nivolumab in relapsed or refractory Hodgkin's lymphoma. $N$ Engl J Med. 2015; 372(4):311-319.

7. Moskowitz CH, Ribrag V, Michot J-M, et al. PD-1 Blockade with the monoclonal antibody pembrolizumab (MK-3475) in patients with classical Hodgkin lymphoma after brentuximab vedotin failure: preliminary results from a phase $1 \mathrm{~b}$ study (KEYNOTE-013). Blood. 2014; 124(21):290.

8. Khoja L, Butler MO, Kang SP, Ebbinghaus S, Joshua AM. Pembrolizumab. J Immunother Cancer. 2015;3:36.

9. Dumaswala K, Mehta A. Novel agents for the treatment of Hodgkin lymphoma. Exp Rev Hematol. 2015;8(5):659-667.

10. Green MR, Monti S, Rodig SJ, et al. Integrative analysis reveals selective 9p24.1 amplification, increased PD-1 ligand expression, and further induction via JAK2 in nodular sclerosing Hodgkin lymphoma and primary mediastinal large B-cell lymphoma. Blood. 2010; 116(17):3268-3277.
11. Armand P. Immune checkpoint blockade in hematologic malignancies. Blood. 2015;125(22):3393-3400.

12. Armand P, Shipp MA, Ribrag V, et al. Programmed death-1 blockade with pembrolizumab in patients with classical Hodgkin lymphoma after brentuximab vedotin failure. J Clin Oncol. Epub 2016 Jun 27.

13. Kwong Y-L, Lopes D, Khong P-L. Low-dose pembrolizumab induced remission in patients with refractory classical Hodgkin lymphoma. Br J Haematol. Epub 2016 Jan 15.

14. Hamid O, Robert C, Daud A, et al. Safety and tumor responses with lambrolizumab (anti-PD-1) in melanoma. N Engl J Med. 2013;369(2): 134-144.

15. Villasboas JC, Ansell SM. Nivolumab for the treatment of classical Hodgkin lymphoma after failure of autologous stem cell transplant and brentuximab. Exp Rev Anticancer Ther. 2016;16(1):5-12.

16. Patnaik A, Kang SP, Rasco D, et al. Phase I study of pembrolizumab (MK-3475; anti-PD-1 monoclonal antibody) in patients with advanced solid tumors. Clinical Cancer Res. 2015;21(19):4286-4293.

17. Robert C, Schachter J, Long GV, et al. Pembrolizumab versus ipilimumab in advanced melanoma. N Engl J Med. 2015;372(26):2521-2532.

18. Pages F, Galon J, Dieu-Nosjean MC, Tartour E, Sautes-Fridman C, Fridman WH. Immune infiltration in human tumors: a prognostic factor that should not be ignored. Oncogene. 2010;29(8):1093-1102.

19. Powles T, Eder JP, Fine GD, et al. MPDL3280A (anti-PD-L1) treatment leads to clinical activity in metastatic bladder cancer. Nature. 2014; 515(7528):558-562.

20. Weber JS, D’Angelo SP, Minor D, et al. Nivolumab versus chemotherapy in patients with advanced melanoma who progressed after anti-CTLA-4 treatment (CheckMate 037): a randomised, controlled, open-label, phase 3 trial. Lancet Oncol. 2015;16(4):375-384.
OncoTargets and Therapy

\section{Publish your work in this journal}

OncoTargets and Therapy is an international, peer-reviewed, open access journal focusing on the pathological basis of all cancers, potential targets for therapy and treatment protocols employed to improve the management of cancer patients. The journal also focuses on the impact of management programs and new therapeutic agents and protocols on

\section{Dovepress}

patient perspectives such as quality of life, adherence and satisfaction. The manuscript management system is completely online and includes a very quick and fair peer-review system, which is all easy to use. Visit http://www.dovepress.com/testimonials.php to read real quotes from published authors. 